The authors are greatly indebted to Professor A M Mancini for his helpful suggestions and criticism. Thanks also to A Busi for the photographic work.

1 Scully R, Mark EJ, McNeely WF. Case records of the Massachussets General Hospital. N Engl F Med 1991;10: 1086-96.

2 Moore JD, Thompson NW, Appelman JD, Foley D. Arteriovenous malformations of the gastrointestinal tract. Arch Sur 1976:111:381-9.

3 Lewi HJE Gledhill T, Gilmour HM, Buist TAS A teriovenous malformations of the intestine. Surg Gynecol teriovenous malformation

4 Fowler DL, Fortin D, Wood WG, Pinkerton JA Jr, Koontz PG Jr. Intestinal vascular malformations. Surgery 1979; PG Jr. Intestir

5 Gallard T. Aneurysmes miliaires de l'estomac, donnant lieu a des hematemeses mortelles. Bull Soc Med Hop Paris $1884 ; 1: 84-91$.

6 Dieulafoy G. Exulceratio simplex: l'intervention chirurgicale dans les hematemeses foudroyantes consecutives a l'ex- ulceration simplex de l'estomac. Bull Acad Med 1989;39: 49-84.

7 Mikò TL, Thomàzy VA. The caliber persistent artery of the stomach: an unifying approach to gastric aneurysm, Dieulafoy's lesion, and submucosal arterial malformation. Hum Pathol 1988;19:914-21.

8 Mortesen NJMcC, Mountford RA, Davies JD, Jeans WD. Dieulafoy's disease: a distinctive arteriovenous malformation causing massive gastric haemorrhage. $\mathrm{Br} \mathcal{F}$ Surg 1983;70:76-8.

9 Veldhuyzen van Zanten SJO, Bartelsman JFWM, Shipper MEI, Tytgat GNJ. Recurrent massive haematemesis from Dieulafoy's vascular malformations-a review of 101 cases. Gut 1986;27:213-22.

10 Grundmann E. Histologic types and possible initial stages in early gastric carcinoma. Beitrage zur Pathologie 1975; 154:256-80.

11 Mower GA, Whitehead R. Gastric hemorrhage due to ruptured arteriovenous malformation (Dieulafoy's disease). Pathology 1986;18:54-7.

12 Jaspersen D, Gaster CB, Koerner TH, Hammar CH. Doppler-controlled injection treatment of Dielafoy's disease. f Gastroenterol Hepatol 1993;8:267-9.

13 Hamrin B. Polymialgia arteritica. Acta Med Scand 1972;7:533.

\title{
Importance of hepatic artery node involvement in patients with colorectal liver metastases
}

\author{
M J Dworkin, S Earlam, C Fordy, T G Allen-Mersh
}

Department of Surgery,

Charing Cross and

Westminster Medical

School, London

M J Dworkin

$S$ Earlam

C Fordy

T G Alllen-Mersh

Correspondence to:

Mr T G Allen-Mersh,

Department of Academic

Surgery,

Chelsea and Westminster

Hospital,

369 Fulham Road,

London SW10 9NH.

Accepted for publication 25 August 1994

\begin{abstract}
Hepatic artery lymph node (HALN) involvement is an adverse prognostic factor in patients treated for colorectal liver metastases. The prevalence of HALN positivity for mid-gut and hind-gut derived colonic tumours, for differing amounts of liver involvement, and for Dukes' $A$ and B versus Dukes' C primary tumours was compared in 75 patients with colorectal liver metastases. All patients whose primary tumours did not invade lymph nodes (Dukes' A or B) had liver metastases that did not involve local hepatic nodes, regardless of the extent of the disease within the liver. This suggests that factors controlling metastasis are not identical with those which control lymphatic invasion in colorectal cancer. HALN positive patients may benefit less from treatment because they are significantly more likely to have both a greater burden of disease within the liver and a tumour with greater lymph invasive potential than patients with HALN negative liver metastases.

(F Clin Pathol 1995;48:270-272)
\end{abstract}

Keywords: Liver metastases, colorectal cancer, hepatic artery lymph node.

Hepatic artery lymph node (HALN) involvement is an adverse prognostic factor in patients being treated by resection ${ }^{1}$ or chemotherapy ${ }^{2}$ for colorectal liver metastases. How- ever, the route and mechanism of HALN involvement is uncertain.

There are two possible routes of involvement. Firstly, by direct extension of disease along the para-aortic lymph nodes from local lymph nodes draining the primary colonic tumour. If this were the case, then the prevalence of HALN positivity would be greater where the primary tumour and associated local nodes were closer to the hepatic artery-that is, primary tumours arising in mid-gut derived colon supplied by the superior mesenteric artery, than if the primary tumour and associated nodes arose more caudally in the hind-gut, which is supplied by the inferior mesenteric artery. ${ }^{3}$ Secondly, HALN involvement could occur as a result of lymphatic spread directly from the haematogenously derived liver metastases into the local lymph nodes draining the liver. ${ }^{4}$

We have examined this by measuring the prevalence of HALN positivity in patients with colorectal liver metastases and correlating this with primary tumour location in the colon and with the extent of liver metastases.

\section{Methods}

Patients with primary colorectal carcinoma and either synchronous or metachronous liver metastases undergoing hepatic artery infusional chemotherapy were prospectively studied. At the time of laparotomy for insertion of a hepatic artery catheter, hepatic artery lymph nodes adjacent to the junction between the common 
Prevalence of HALN involvement by primary tumour node status and location, and extent of liver involvement by HALN status. Although colonic tumours which had not involved primary nodes produced liver metastases, it can be seen that these metastases never involved hepatic artery lymph nodes.

\begin{tabular}{lclc}
\hline HALN involvement & No. of patients & \multicolumn{2}{l}{ Hepatic artery node } \\
\cline { 3 - 4 } & & Negative & Positive \\
\hline $\begin{array}{l}\text { Primary tumour node } \\
\quad \text { negative }\end{array}$ & 17 & 17 & 0 \\
$\begin{array}{l}\text { positive } \\
\quad \text { mid-gut }\end{array}$ & 58 & 37 & 21 \\
$\quad$ hind-gut & 9 & 6 & 3 \\
Extent of liver metastases* & 56 & 41 & 15 \\
& 68 & $18 \cdot 0(16 \cdot 1 \%)$ & $28 \cdot 7(18 \cdot 0 \%)$ \\
\hline
\end{tabular}

* Expressed as mean (SD) PHR.

hepatic artery and gastroduodenal artery were excised for routine histopathological examination.

The extent of liver metastases was assessed from a computed tomography (CT) scan carried out before surgery. The area of liver and tumour outlined on each CT scan was estimated using a point counting technique. The ratio of tumour to total liver area was then translated into volume by Delesse's ${ }^{5}$ principle and the percentage hepatic replacement (PHR) was calculated.

The primary tumour was routinely excised with the regional lymph node pedicle. This was then examined histologically for evidence of lymph node involvement. The location of the tumour was noted. Tumours arising from the embryological mid-gut (proximal to and including the splenic flexure) were regarded as having lymph node drainage via the superior mesenteric artery; those arising from the hindgut (distal to the splenic flexure) were regarded as draining into the inferior mesenteric arterial lymph node group.

Statistical analysis of the significance of the differences between HALN status, primary tumour lymph node status and tumour location were assessed by contingency table analysis using the $\chi^{2}$ test. Differences in PHR were assessed using the Student's $t$ test with confidence interval (CI) analysis.

\section{Results}

Seventy five patients were studied but as all variables were not available for all patients, the totals vary slightly for each analysis (table).

The prevalence of HALN positivity was not significantly different in liver metastases from tumours arising in mid-gut derived colon (three of nine; $33.3 \%$ ) compared with those arising from hind-gut derived colonic tumours (14 of $56 ; 25 \cdot 0 \%)$. There was a significant $(t=2 \cdot 047$, $\mathrm{p}=0.02)$ difference $(95 \%$ CI $19 \cdot 3-0.243 \%)$ in the PHR of HALN positive compared with HALN negative liver metastases.

There was a significant $(p<0.01)$ difference in the prevalence of HALN involvement in patients with primary tumour node negative (none of $17 ; 0 \%$ ) compared with those with primary tumour node positive (21 of 58 ; $36 \cdot 2 \%$ ) liver metastases. Among those with primary tumour node positive metastases, there was a significant difference $(t=2 \cdot 307, \mathrm{p}=0.01)$
(95\% CI $21 \cdot 0-1 \cdot 45 \%$ ) in the PHR of HALN positive (mean (SD) $28.8 \%(17 \cdot 5 \%)$ ) compared with HALN negative (17.5\% (15.6\%)) liver metastases.

\section{Discussion}

We did not find a significant increase in the prevalence of HALN involvement with liver metastases arising from mid-gut compared with hind-gut derived colonic tumours. This does not support the hypothesis that HALN involvement occurs by nodal spread from primary tumour colonic nodes via para-aortic and coeliac axis nodes to the hepatic artery nodes. However, there was significantly more disease within the liver in HALN positive than in HALN negative patients. This is more consistent with the view that hepatic node involvement results predominantly from spread of malignant cells within liver metastases into the lymph nodes which drain the liver. ${ }^{4} \mathrm{Al}$ though there was a significant correlation between the extent of liver disease and HALN involvement, there was wide variation in the extent of liver involvement in both HALN positive and negative groups (table). This suggests that factors other than extent of liver metastases also influence HALN involvement.

In this study all patients whose primary tumours did not invade local lymph nodes (Dukes' A or B) had liver metastases which did not invade nodes, regardless of the extent of disease within the liver (table). Thus, despite the primary tumour's ability to metastasise to the liver, lymphatic spread did not occur in these patients. A previous study, ${ }^{4}$ involving a retrospective review of HALN positivity in five patients with colorectal liver metastases, reported HALN involvement in three patients with primary node negative tumours. It is not clear why their experience is different to ours, but one factor might be the accuracy of node staging in a retrospective study.

Lymphatic spread from haematogenously derived metastases provides an unusual natural experiment where the tumour's capabilities for metastasis and for lymphatic invasion are separated. The results of this experiment in the present study suggest that these capabilities do not exactly coincide. It appears that the factors controlling metastasis are not identical with those controlling lymphatic invasion in colorectal cancer.

When the bias resulting from differences in tumour lymph invasive potential was reduced by analysing primary tumour node positive (Dukes' C) tumours only, the influence of the extent of liver disease involvement was stronger. Thus, although the results suggested that HALNs were usually involved as a result of direct spread via lymphatic vessels from the hepatic tumour, this only occurred if the primary tumour was lymph invasive.

HALN positive patients benefit less from liver resection ${ }^{1}$ and hepatic artery infusion chemotherapy ${ }^{2}$ than HALN negative ones. The results in this study suggest that this is because HALN positive patients are more likely to have a greater burden of disease within the liver and 
a more lymph invasive tumour compared with those with HALN negative liver metastases.

We are indebted to the Cancer Relief Macmillan fund for we are of MJD.

1 Ekberg H, Kranberg KJ, Anderson R, Lundstedt C, Hagerstrand I, Ranstam J, et al. Pattern of recurrence in liver resection for colorectal secondaries. World $\mathcal{F}$ Surg 1987;11: $541-7$.
2 Chang AE, Schneider PD, Sugarbaker PH, Simpson C Culane $M$, Steinberg SM. A prospective randomised tria of regional versus systemic continuous FUDR chemotherapy in the treatment of colorectal liver metastases. An urg 1987;206:685-93.

3 Last JR. Lymph drainage of the alimentary canal. In: Anatomy regional and applied. 4th edn. London: J \& A Churchill, 1966:425-6.

4 August DA, Sugarbaker PH, Schneider PD. Lymphatic dissemination of hepatic metastases. Cancer 1985;55:1490-4.

Delesse A. Procede mechanique pour determines la composition des roches [extrait]. C R Acad Sci (Paris) 1847, 25.544-7.

\title{
An unusual case of colonic angiodysplasia
}

\author{
N J Trendell-Smith, B F Warren, E A Sheffield, P Durdey
}

\begin{abstract}
An unusual case of a colonic vascular anomaly resembling angiodysplasia associated with right sided diverticular disease is presented. The patient, a 74 year old man, presented with a four day history of rectal bleeding and subsequently underwent hemicolectomy. The resected specimen was flushed out with heparin-saline solution and injected with $a$ bariumgelatine mixture. Preoperative barium enema revealed right sided diverticula, whereas post-resection angioradiography revealed the "coral reef" vascular anomaly consistent with angiodysplasia. Histology confirmed the presence of both diverticular disease and angiodysplasia. This case report highlights the importance of considering a vascular anomaly in patients presenting with rectal bleeding despite the presence of another radiologically demonstrable anatomical lesion.
\end{abstract}

(F Clin Pathol 1995;48:272-275)

Keywords: Angiodysplasia, diverticular disease, vascular anomaly.

Department of Histopathology, Bristol Royal Infirmary, Marlborough Street, Bristol BS2 8HW N J Trendell-Smith.

B F Warren

E A Sheffield

Department of Surgery,

Bristol Royal

Infirmary

P Durdey

Correspondence to: Dr N J Trendell-Smith.

Accepted for publication 7 June 1994 elderly, ${ }^{23}$ but only a minority of patients presen with prolonged lower intestinal bleeding.

Although angiodysplasia is regarded as a separate vascular entity, its classification and differentiation from other vascular conditions still presents a clinicopathological problem. Much of this relates to our poor understanding of the pathogenesis of angiodysplasia. Several clinical associations have been described. These include aortic stenosis (more precisely the association of rectal bleeding with aortic stenosis), ${ }^{5}$ chronic renal failure and cirrhosis. ${ }^{6}$ Angiodysplasia is currently thought to represent a degenerative vascular change with the histological hallmark of mucosal vascular ectasia. ${ }^{4}$ However, cases have been described in the young, questioning this degenerative pathogenesis. $^{7}$

Here, we present a case in which an unusual form of angiodysplasia occurred in association with congenital right sided diverticular disease. This dual pathology highlights the importance of considering a vascular abnormality of the intestine as a cause of rectal bleeding despite the presence of another radiologically demonstrable anatomical lesion.

\section{Case report}

A 74 year old white man presented with a four day history of rectal bleeding, passing stools mixed with fresh, bright red blood. He was otherwise well with no other gastrointestinal symptoms. He had previously had a villous adenoma of the rectum resected endoscopically three years before. On examination, he was haemodynamically stable with a haemoglobin concentration of $13.9 \mathrm{~g} / \mathrm{dl}$ and a precordial ejection systolic murmur that radiated into the axilla, interpreted as aortic stenosis. There was no evidence of circumoral or gingival telangiectasia and no family or past medical history of rectal bleeding, telangiectasia, epistaxis, or haemoptysis. There were no signs of congestive cardiac failure, renal insufficiency, portal hypertension, or varices. 